\title{
Traumatic Hemipelvectomy with Free Gluteus Maximus Fillet Flap Covers: A Case Report
}

\author{
WI Faisham, MMed Orth, WS Azman*, MS Plastic (USM), TMS Muzaffar, MMed Orth, DAJ Muslim, \\ MRCS, AH Azhar**, MMed Surg (USM), MM Yahya**, MMed Surg (USM) \\ Department of Orthopedics, Universiti Sains Malaysia, Kota Bahru, Malaysia \\ *Plastic and Reconstructive Unit, Universiti Sains Malaysia, Kota Bahru, Malaysia \\ **Department of Surgery, Universiti Sains Malaysia, Kota Bahru, Malaysia
}

\begin{abstract}
Traumatic hemipelvectomy is an uncommon and life threatening injury. We report a case of a 16-year-old boy involved in a traffic accident who presented with an almost circumferential pelvic wound with wide diastasis of the right sacroiliac joint and symphysis pubis. The injury was associated with complete avulsion of external and internal iliac vessels as well as the femoral and sciatic nerves. He also had ipsilateral open comminuted fractures of the femur and tibia. Emergency debridement and completion of amputation with preservation of the posterior gluteal flap and primary anastomosis of the inferior gluteal vessels to the internal iliac artery stump were performed. A free fillet flap was used to close the massive exposed area.
\end{abstract}

Key Words:

traumatic hemipelvectomy, amputation, and free gluteus maximus fillet flap

\section{INTRODUCTION}

Traumatic hemipelvectomy is a life threatening injury that requires rapid resuscitation, early surgical intervention and intensive care monitoring. Improvements in early surgical care have resulted in an increase in the survival rate following this devastating injury ${ }^{1}$. Controversies exist regarding whether to close or leave the primary amputation wound open and the method of soft tissue covering ${ }^{2}$. This case highlights a complex injury of hemipelvectomy whereby emergency completion of amputation followed by re-vascularisation of the gluteus flap to cover the massive defect in a single stage surgery.

\section{CASE REPORT}

A 16-year-old male pillion rider on a motorcycle was involved in a head-on collision with a car at a speed of 100 $\mathrm{km} / \mathrm{h}$. He was brought immediately to a primary care hospital where initial resuscitation with fluids and transfusion of two pints of blood took place; he was transferred to our centre within an hour. Upon arrival, blood pressure was recorded at $90 / 55 \mathrm{~mm} / \mathrm{hg}$ with a heart rate of 110 bpm. Pelvic instability was stabilized with tight Hammock slings and he was transfused another two pints of blood and four units of plasma and cryoprecipitate at which point he was haemodynamically stable.

There was a large circumferential laceration wound over the iliac crest extending to the perineum and posterior sacrum with a skin paddle of $6 \mathrm{~cm}$ from the sacrum (Figure 1a). The wound was badly contaminated and the iliopsoas muscle was badly contused. The external and internal iliac vessels were avulsed and the common iliac vessels were thrombosed. Both the sciatic and femoral nerves were avulsed from their roots. The anus was lacerated and the anal sphincter was avulsed. The peritoneum was exposed and badly contaminated but not breeched. There was no injury to the bladder, bowel or right ureter. Both the sacroiliac joint and symphysis pubis were widely diastatic and grossly unstable (Figure 1b) . There was minimal bleeding from the wound edge and the sacroiliac plexus as all vessels were already thrombosed (Figure 1c).

The ipsilateral thigh had a comminuted fracture at the shaft with a $10 \mathrm{~cm}$ laceration wound with circumferential degloving of the skin and muscle contusion. There was also a closed mid-shaft fracture of the tibia with moderate soft tissue contusion. Radiographs revealed wide diastasis of the symphysis pubis and sacroiliac joint and computed tomography (CT) scan with contrast revealed a thrombosed external iliac artery (Figure $1 \mathrm{~b}$ and $1 \mathrm{c}$ ).

The patient underwent emergency debridement with pulsatile lavage and hemipelvectomy due to avulsion of major lower limb neurovascular structures and associated gross contamination. The soft tissue defect over the pelvis was covered with a gluteus maximus fillet flap while maintaining the skin paddle over the sacrum. Vascularity of the gluteus maximus was reestablished by 8 hours after the 


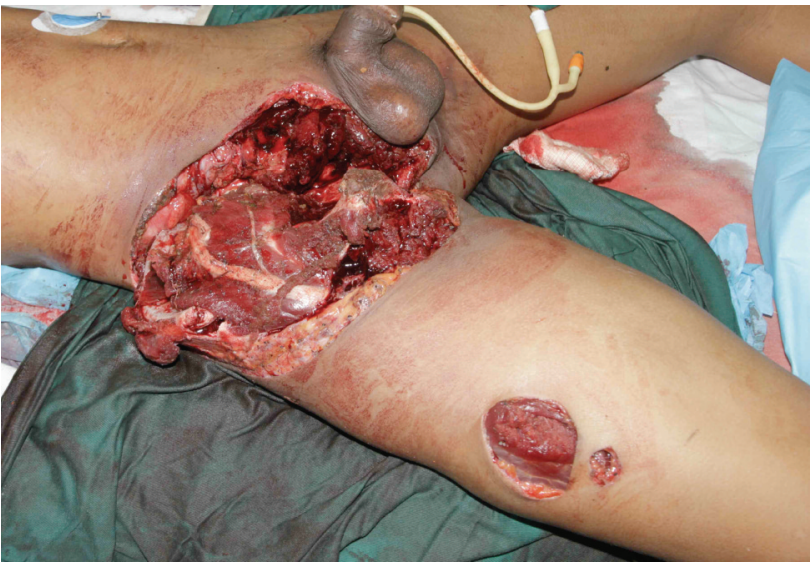

Fig. 1a: Anterior view of traumatic right hemipelvectomy wound.

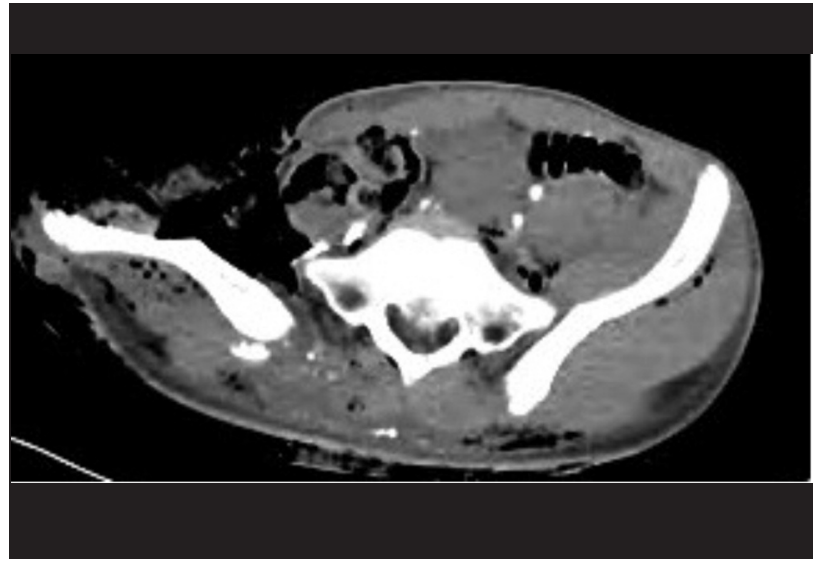

Fig. 1 b: Plain radiograph showed wide diastasis of symphysis pubis and separation of right sacroiliac joint.



Fig. 1c: Computed Tomography (CT imaging) revealed thrombosed external iliac artery. A 3D reconstruction image showed vessels thrombosed at the common iliac artery.

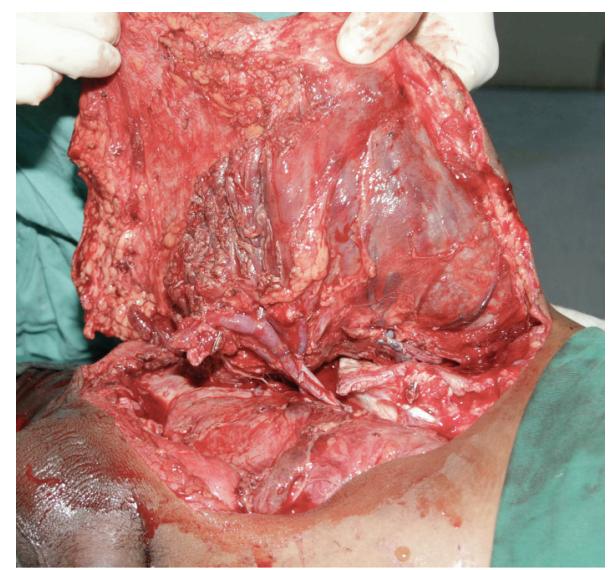

Fig 2a: Intra-operative photograph post anastomosis of inferior gluteal artery to external iliac artery of gluteus maximus flap.

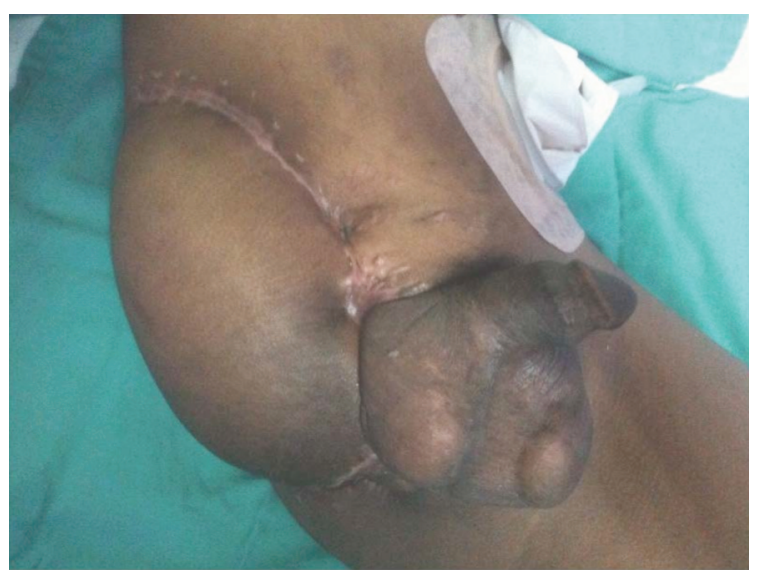

Fig 2b: A completely healed traumatic hemipelvectomy wound covered with free gluteus maximus fillet flap 
injury by anastomosing the remnant of the inferior gluteal artery and vein to the external iliac vessels. A right transverse colostomy was performed for wound care of the perineal and anal laceration. Second look surgery and debridement of devitalized gluteus maximus muscle was performed twice. The flap survived, although complications secondary to infection required prolonged intravenous antibiotics and wound care.

Secondary closure of the wound was performed on the 26th post-operative day. The patient was prescribed extensive rehabilitation for sitting and mobilisation. Upon discharge he was able to ambulate independently with double crutches. Follow up after 2 months of initial injury showed the wound had healed well (Figure 2b).

\section{DISCUSSION}

Traumatic hindquarter amputation with complete disruption through symphysis pubis and sacroiliac joint is a very rare injury. Most survivors of traumatic hemipelvectomy are young, healthy individuals, who are able to tolerate massive haemorrhage and soft tissue destructions ${ }^{1,2}$. Early and vigorous resuscitation and vascular control is a major determinant of survival ${ }^{1,2}$.

Patients with complete hemipelvectomy seem to have a higher survival rate compared to those with partial hemipelvectomy. Complete transection of the vessels allows immediate thrombosis and subsequent control of haemorrhage and leads to thrombosis of the sacroiliac venous plexus. These findings were observed in our patient as minimal bleeding occurred at the sacroiliac plexus and all internal and external vessels were indeed thrombosed with minimal active bleeding. Persistent hypotension can play a part in preventing dislodging of thrombi from the main vessels.

Initial coverage of the wound is desirable to protect the exposed structures ${ }^{1,2,3}$, prevent infection and facilitate early patient rehabilitation. Several options are available for coverage, including a posterior or anterior flap using gluteus and anterior thigh compartment muscle and a vertical rectus abdominis myocutaneous flap ${ }^{3}$. Use of a free flap using latissimus dorsi muscle or limb fillet harvested from the thigh or leg is an option for secondary wound closure ${ }^{3}$. There is controversy regarding which are the best flaps to cover such massive defects especially during emergency procedures for trauma cases. The present case highlighted that primary wound coverage with a vascularized flap is a viable option as infection was controlled and the wound healed. Vacuum-assisted closure (VAC) has been suggested for temporary coverage in the management of large tissue defects in the pelvic regions. VAC also supports wound conditioning, reduces infectious complications and facilitates definitive wound closure ${ }^{4}$.

Hemodynamic stability and speed of surgery as well as flap reliability should be taken in consideration for management of this rare injury. We chose to useposterior gluteal flap as there was no other viable option left since the patient had fractures of both femurs and tibiae with accompanying soft tissue injury. Early re-vascularisation of the gluteus maximus by re-anastomosis of the inferior gluteal vessel to the remnant of external iliac artery resulted in maintenance of flap viability.

\section{REFERENCES}

1. Lasurt S, Combalia-Aleu A. Complete traumatic hemipelvectomy: Report of a case after 19 years of follow-up. Injury. 2010; 41(8): 874-82.

2. Schoderbek RJ, Battaglia TC, Dorf ER, Kahler DM. Traumatic hemipelvectomy: a case report and literature review. Arch Orthop. Trauma Surg. 2005; 125(5): 538-62.

3. AZ Mat Saad, AS Halim, WI Faisham, WS Azman, W Zulmi. Soft tissue reconstruction following hemipelvectomy: Eight year experience and literature review; Scientific World Journal 2012: 702904, Epub 2012 May 2, available at http://www.tswj.com/2012/702904/

4. Labler L, Trentz O. The use of vacuum-assisted-closure (VAC) in soft tissue injuries after high-energy pelvic trauma. Langenbecks Arch Surg 2007; 392(5): 601-9. 